\title{
The Work of the 2014 Fields Medalists
}

Welington de Melo, Bjorn Poonen, Jeremy Quastel, and Anton Zorich

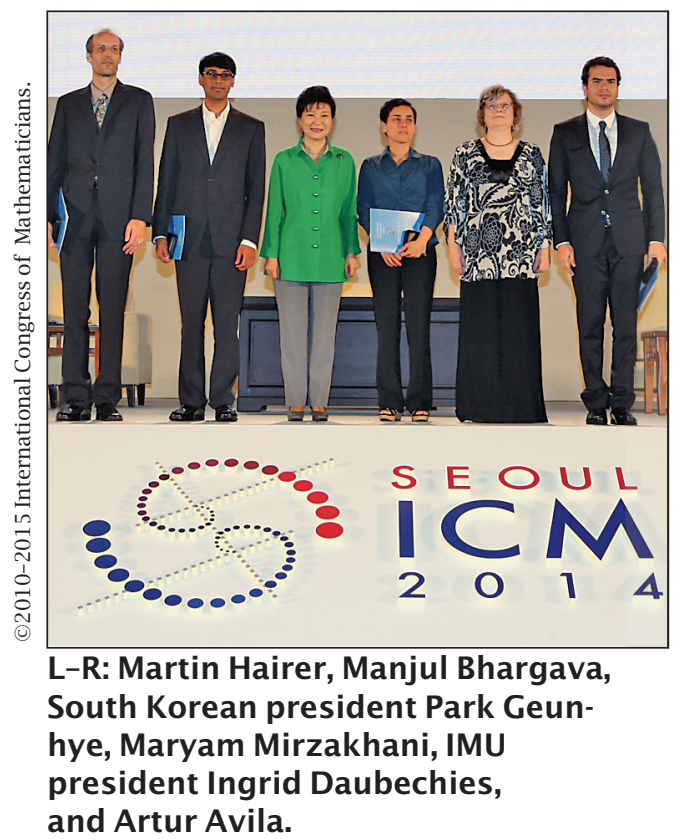

The Notices solicited the following articles about the works of the four individuals to whom Fields Medals were awarded at the International Congress of Mathematicians in Seoul, South Korea, in August 2014. This was a historic occasion, as it marked the first time since the medal was established in 1936 that a woman was among the recipients. The International Mathematical Union also issued news releases describing the medalists' work, and these appeared in the October 2014 issue of the Notices.

- Allyn Jackson 


\section{Welington de Melo}

\section{The Work of Artur Avila}

Artur Avila was awarded the Fields Medal in 2014 for his deep contributions to dynamical systems and to the spectral theory of one-frequency Schrödinger operators. Many of his profound achievements -in one-dimensional dynamics, both real and complex, as well as in flat billiards and in the spectral theory of Schrödinger operators-are characterized by an intense use of the powerful ideas of renormalization. He has also made deep advances in the theory of conservative dynamical systems in any dimension and in the stable ergodicity of partially hyperbolic systems.

Artur Avila was born in Rio de Janeiro, Brazil, where he lived until he finished his PhD studies at the Instituto de Matemática Pura e Aplicada (IMPA) in 2001. Before starting his postdoc position in the Collège de France in Paris, Artur, with collaborators, obtained a complete description of typical dynamics of unimodal interval maps, i.e., smooth interval maps with a unique nonflat critical point. It was already known that the space of unimodal maps contains two disjoint regions where the dynamics is well understood. For maps in the regular region, there exists a unique attracting fixed point, and the trajectories of almost all initial conditions, in the Lebesgue measure sense, are asymptotic to this periodic point. The typical dynamical behavior is therefore periodic. For maps in the stochastic region the dynamics is chaotic but with a good statistical description: there exists an absolutely continuous invariant measure that controls the dynamics of a typical orbit in the sense that the time average of any physical observable is equal to the space average of the observable with respect to this measure. In particular, the frequency of visits of a typical orbit to any interval is equal to the measure of this interval. In the complement of these two regions there are maps where the dynamics can be completely described but there are other maps whose dynamics is pathological. These different dynamical behaviors were already present in the famous quadratic family $x \in[0,1] \mapsto q_{\mu}=\mu x(1-x)$, where the parameter $\mu$ belongs to the interval $(0,4]$. The set of parameter values corresponding to regular maps is open and dense by a difficult result proved in [18] and [20]. On the other hand, the set of parameter values corresponding to stochastic maps has positive Lebesgue measure, as proved in [20], and the complement has measure zero [22] but positive Hausdorff dimension. The main result in [5], [9]

W. de Melo is a professor at the Instituto de Matemática Pura e Aplicada. His email address is deme1o . impa@gmai 1 . com.

For permission to reprint this article, please contact: reprint-permission@ams .org.

DOI: http://dx.doi.org/10.1090/noti1317 is that, for typical one-dimensional families of unimodal maps, one does not see the third region; i.e., the set of parameter values corresponding to maps in the third region has Lebesgue measure zero. For a typical one-parameter family of unimodal maps we have a decomposition of the parameter space with the same properties as the quadratic family.

Another important result in his early career is the unexpected rigidity in the space of unimodal maps obtained in [10]. In this article it is shown that there is a large set $R$ of unimodal maps such that any typical one-parameter family intersects $R$ in a set of parameters of full Lebesgue measure in the complement of the regular parameters and any two maps in $R$ that are topologically conjugate are in fact smoothly conjugate. In particular, the authors provide a combinatorial formula to calculate the multipliers of all periodic points for maps in $R$.

More recently, in complex one-dimension dynamics, Avila and Lyubich described several fundamental properties on the geometry of the Julia set of Feigenbaum-type quadratic polynomials [6], [7], [2], [3], [4]. In particular, the existence of examples of such quadratic polynomials with Julia sets of positive Lebesgue measure is proven.

In most of these results, Avila uses as a fundamental tool the renormalization theory. This theory started with experimental discovery by the physicists Feigenbaum and Coullet-Tresser in the 1970s on the transition from simple to chaotic dynamics in families of unimodal maps. They formulated a conjecture that involves a nonlinear operator in the space of unimodal maps. The proof of the conjecture and some generalizations involve the work of several mathematicians such as Sullivan, McMullen, and Lyubich. Finally, Avila and Lyubich in [16] gave a very conceptual and much simpler proof of the general conjecture that holds also in the space of unimodal maps with higher criticality.

A second area of dynamical systems where Avila made fundamental contributions is the dynamics of interval exchange transformations, regular polygonal billiards, and ergodic properties of the geodesic Teichmüller flow in the moduli space of Abelian differentials on Riemann surfaces.

Given a partition $I_{1}, \ldots, I_{n}$ of the interval $[0,1]$ in $d \geq 2$ intervals and a permutation $\sigma$ of $\{1, \ldots, d\}$, we can define the mapping $T:[0,1] \rightarrow[0,1]$ by

$$
T(x)=x-\sum_{j<i} \lambda_{j}+\sum_{\sigma(j)<\sigma(i)} \lambda_{j}
$$

if $x \in I_{i}$, where $\lambda_{j}$ is the length of the interval $I_{j}$. This is what is called an interval exchange transformation, and it is completely characterized by the permutation $\sigma$ and by the vector $\left(\lambda_{1}, \ldots, \lambda_{d}\right)$ that belongs to a simplex in $\mathbb{R}_{+}^{d}$. The space of such maps is therefore finite dimensional. We say that a subset is typical if the complement has 
zero Lebesgue measure. Veech proved that for any irreducible permutation the typical interval exchange transformation is uniquely ergodic. In 1980 Katok proved that no interval exchange transformation is mixing. Recall that a measurepreserving transformation $f:(X, \mu) \rightarrow(X, \mu)$ is mixing if

$$
\lim _{\mathrm{n} \rightarrow \infty}\left[\mu\left(\mathrm{f}^{-\mathrm{n}}(\mathrm{A}) \cap \mathrm{B}-\mu(\mathrm{A}) \mu(\mathrm{B})\right]=0\right.
$$

for any measurable sets $A$ and $B$, and it is weak mixing if

$$
\lim _{\mathrm{N} \rightarrow \infty} \frac{1}{\mathrm{~N}} \sum_{\mathrm{n}=0}^{\mathrm{N}}\left[\mu\left(\mathrm{f}^{-\mathrm{n}}(\mathrm{A}) \cap \mathrm{B}-\mu(\mathrm{A}) \mu(\mathrm{B})\right]=0 .\right.
$$

The joint work of Avila and Forni [11] solved the main problem in the ergodic theory of interval exchange transformation: a typical interval exchange transformation is weakly mixing for any irreducible permutation that is not a rotation. This work is connected to a recent result of Avila and Delecroix proving that almost all regular polygonal billiards are weakly mixing. Again, one of the main tools for these results is a renormalization operator that consists of considering the first return map to a smaller interval and rescaling to the original size. It is called the Rauzy-Veech renormalization operator. Veech and Masur proved that it has an absolutely continuous invariant measure, and the ergodic properties of the operator give important information about the ergodic properties of a typical interval exchange transformation. The invariant measure is not finite, but in [25] Zorich defined an accelerated version of the renormalization operator, called the Rauzy-Veech-Zorich operator, that does have a finite absolutely continuous invariant measure. The new operator maps each interval exchange transformation into an iterate of the previous operator, the iterate depending on the interval exchange transformation. The dynamics of this new operator is closely related to the so-called Teichmüller geodesic flow that acts in the moduli space of Abelian differentials in compact Riemann surfaces of a given genus. Zorich experimentally discovered the existence of $d$ exponents that for a typical interval exchange transformation with $d$ intervals describes the deviation of the ergodic average from the mean and found that these exponents are given by the Lyapunov spectrum of the so-called Rauzy-Veech-Zorich cocycle over the renormalization operator. In [12] Avila and Viana proved the Kontsevich-Zorich conjecture stating that the Lyapunov spectrum of the Rauzy-VeechZorich cocycle is simple. Another important result in this area was obtained by Avila and collaborators establishing the exponential decay of correlation of the Teichmüller flow on strata of the moduli space of Abelian differentials that was conjectured by Veech.

The area of one-frequency Schrödinger operators was very active before the arrival of Avila. In particular, it was known that if one multiplies an analytic potential by a coupling constant generating a one-parameter family of operators, then for very small values of the coupling constant the spectrum of the operator is absolutely continuous, and for very large values the spectrum is pure point for typical values of the frequency. Not much was known for values of the coupling constant in between. However, it was known that to an operator corresponds a one-parameter family of $S L(2, \mathbb{R})$ cocycles, parametrized by the energy, and it was known that the spectrum coincides with the bifurcation set of the dynamical object. These are the values of the parameter such that the cocycle is not uniformly hyperbolic. Probably inspired by the results in one-dimensional dynamics, Avila described three regions in this space of cocycles: UR (uniform hyperbolic), SpC (supercritical), and SbC (subcritical) and called the complement of these $\mathrm{C}$ (critical). The cocycles in SpC are not uniformly hyperbolic but have positive Lyapunov exponents and were already well understood. The part of the spectrum of the operator that lies in this region corresponds typically to the pure point spectrum. Avila proved that the part of the spectrum that lies in $\mathrm{SbC}$ corresponds to the absolutely continuous part of the spectral measure. Finally, he proved the existence of a stratification of the space into submanifolds of positive codimension and that the set of critical cocycles is a small subset, in the measure-theoretic sense, of these submanifolds [23]. As a consequence, Avila proved that a typical one-parameter family of cocycles does not contain critical cocycles at all. Also as a consequence of Avila's construction, the spectrum of an operator corresponding to a typical potential, in a measure theoretical-sense (prevalence), decomposes into a finite number of disjoint open sets, and the spectral measure is absolutely continuous or pure point on a given open set if and only if the Lyapunov exponent is zero or positive in this region. This result justifies the intuition from physics that typically one is either in the conductor regime (absolutely continuous spectrum) or the insulator regime (pure point spectrum).

An intensely studied family of Schrödinger operators is the family of almost Mathieu operators, which is related to a celebrated physical phenomena, the quantum Hall effect. This family is not typical in the above sense because it has a critical cocycle where the coupling constant is equal to one. This is forced by a symmetry, the Aubry duality. Before the arrival of Avila in this area, much was known about this family. For example, for almost all frequencies and phases the spectrum is absolutely continuous in the subcritical region (coupling constant smaller than one) and pure point in the supercritical region. Also, the spectrum has positive Lebesgue measure in both regions. Still, some important questions remained open. Avila solved all the 
remaining questions about the spectral measure of the almost Mathieu system: the spectrum is a Cantor set for critical values of the coupling constant for all irrational frequencies [14]; it has zero Lebesgue measure [24]; and for all irrational frequencies and almost all phases, the spectrum is continuous singular. To summarize, one can now say with Avila that the spectral measure is absolutely continuous precisely in the subcritical case and pure point precisely in the supercritical case.

In another area of dynamical systems, Avila proved that a $C^{1}$ volume-preserving diffeomorphism of a compact manifold can be approximated in the $C^{1}$ topology by a $C^{\infty}$ volume-preserving diffeomorphism [1]. This was an open question for more than thirty years, and Avila's proof involves solving a partial differential equation with low regularity. The result is very relevant for the description of generic properties of conservative dynamical systems, because another fundamental tool, the closing lemma, is known only in the $C^{1}$ topology, and for good distortion estimates one needs higher regularity.

\section{References}

[1] A. AvILA, On the regularization of conservative maps, Acta Math. 205 (2010), 5-18.

[2] A. AvilA and M. LyUbich, Examples of Feigenbaum Julia sets with small Hausdorff dimension, in Dynamics on the Riemann Sphere (eds. P. Hjorth and C. Peterson), European Math. Soc., Zürich, 2006, pp. 71-87.

[3] _ Hausdorff dimension and conformal measures of Feigenbaum Julia sets, J. Amer. Math. Soc. 21 (2008), 305-383.

[4] _ Feigenbaum Julia sets of positive area, manuscript, 2011.

[5] A. AvilA, M. Lyubich and W. DE Melo, Regular or stochastic dynamics in real analytic families of unimodal maps, Invent. Math. 154 (2003), 451-550.

[6] A. Avila, J. KAHn, M. Lyubich, and W. Shen, Combinatorial rigidity for unicritical polynomials, Annals of Math. (2) 170 (2009), 783-797.

[7] A. Avila, M. Lyubich, and W. Shen, Parapuzzle of the Multibrot set and typical dynamics of unimodal maps, $J$. European Math. Soc. 13 (2011), 27-56.

[8] A. Avila and C. G. MoreIRA, Statistical properties of unimodal maps: The quadratic family, Annals of Math. (2) 161 (2005), 831-881.

[9] _ Statistical properties of unimodal maps: Smooth families with negative Schwarzian derivative, Geometric Methods in Dynamics, I, Astèrisque 286 (2003), 81-118.

[10] _ Statistical properties of unimodal maps: Physical measures, periodic orbits and pathological laminations, Publ. Math. IHÉS 101 (2005), 1-67.

[11] A. AvILA and G. ForNI, Weak mixing for interval exchange transformations and translation flows, Annals of Math. 165 (2007), 637-664.

[12] A. Avila and M. VIANA, Simplicity of Lyapunov spectra: Proof of the Zorich-Kontsevich conjecture, Acta Math. 198 (2007), 1-56.

[13] A. AVILA and C. G. MoreIRA, Hausdorff dimension and the quadratic family, manuscript, 2002.
[14] A. Avila and Svetlana Jitomirskaya, The Ten Martini Problem, Annals of Math. 170 (2009), 303-342.

[15] A. AvILA, On the regularization of conservative maps, Acta Math. 205 (2010), 5-18.

[16] A. Avila and M. LyUbich, The full renormalization horseshoe for unimodal maps of higher degree: Exponential contraction along hybrid classes, Publ. Math. IHÉS 114 (2011), 171-223.

[17] A. Avila and S. GouÈzel, Small eigenvalues of the Laplacian for algebraic measures in moduli space, and mixing properties of the Teichmüller flow, Annals of Math. 178 (2013), 385-442.

[18] J. GRACZYK and G. SWIATEK, Generic hyperbolicity in the logistic family, Annals of Math. (2) 146 (1997), 1-52.

[19] M. JAKOBSON, Absolutely continuous invariant measures for one-parameter families of one-dimensional maps, Comm. Math. Phys. 81 (1981), 39-88.

[20] M. LyUBICH, Dynamics of quadratic polynomials I, II, Acta Math. 178 (1997), 185-247, 247-297.

[21]_, Feigenbaum-Coullet-Tresser universality and Milnor's hairiness conjecture, Annals of Math. (2) 149 (1999), 319-420.

[22] _ Almost every real quadratic map is either regular or stochastic, Annals of Math. (2) 156 (2002), 1-78. [23] A. AvILA, Global theory of one-frequency Schrod̈inger operators I, II, preprint, 2009.

[24] A. AVILA and R. KRIKORIAN, Reducibility or uniform hyperbolicity for quasiperiodic Schrödinger cocycles, Ann. of Math. (2) 164 (2006), 311-940.

[25] A. ZorICH, Finite Gauss measure on the space of interval exchange transformations. Lyanpunov exponents, Ann. Inst. Fourier (Grenoble) 46 (1996), 325-370.

\section{Bjorn Poonen}

\section{The Work of Manjul Bhargava}

Anyone solving equations over $\mathbb{Z}$ or $\mathbb{Q}$ soon recognizes the need to study the arithmetic of higher number fields and algebraic varieties. Manjul Bhargava has found new and improved ways to count many such objects by understanding the orbits of $G(\mathbb{Z})$ on $V(\mathbb{Z}):=\mathbb{Z}^{n}$ for various representations of algebraic groups $G \rightarrow \mathrm{GL}(V)$ over $\mathbb{Z}$. This has led to dramatic consequences in arithmetic geometry concerning the average behavior of elliptic curves and higher-genus curves over $\mathbb{Q}$.

\section{Counting Number Fields}

Consider the problem of estimating the number of isomorphism classes of degree $n$ number fields $k$ whose absolute discriminant $D_{k}$ satisfies $\left|D_{k}\right| \leq X$ as $X \rightarrow \infty$. The primitive element theorem guarantees that each such $k$ is obtained by adjoining a root of a degree $n$ irreducible polynomial over $\mathbb{Q}$. But there are many polynomials giving rise to each number field, and eliminating the redundancy is tricky.

Bjorn Poonen is professor of mathematics at the Massachusetts Institute of Technology. His email address is poonen@math . mit.edu. 
One can attempt to solve this and other arithmetic counting problems by following the outline below:

(1) Identify a representation $G \rightarrow \mathrm{GL}(V)$ and local conditions (inequalities and congruence conditions) such that the nondegenerate $G(\mathbb{Z})$-orbits in $V(\mathbb{Z})$ satisfying the local conditions parametrize the objects of interest.

(2) Count the orbits satisfying the inequalities by counting lattice points inside a fundamental domain (a region in $V(\mathbb{R})$ consisting of one representative of each $G(\mathbb{Z})$-orbit).

(3) Sieve out the orbits that violate the congruence conditions.

Davenport and Heilbronn [19] successfully carried out this three-step approach to count degree 3 number fields of bounded discriminant (and they were preceded by Gauss, who studied the average behavior of class numbers of quadratic fields, possibly by a similar method). As will be explained, Bhargava has contributed significant ideas improving all three steps, enabling him to carry out this approach in much more complicated situations.

Consider Step 1. Davenport and Heilbronn used a bijection taking certain $\mathrm{GL}_{2}(\mathbb{Z})$-orbits in the space $\mathrm{Sym}^{3} \mathbb{Z}^{2}$ of degree 3 homogeneous forms over $\mathbb{Z}$ to degree 3 number fields. For $n \geq 4$, however, the representation $S_{y m}^{n} \mathbb{Z}^{2}$ of $\mathrm{GL}_{2}$ does not yield a similar parametrization of degree $n$ number fields. Instead, one studies prehomogeneous vector spaces, vector spaces $V$ equipped with an algebraic group action for which geometrically there is a Zariski dense orbit. (The Zariski dense orbit condition is natural in hindsight, since any two degree $n$ number fields become isomorphic when tensored with $\mathbb{C}$.) Prehomogeneous vector spaces were classified in [27]. Wright and Yukie carried out a detailed study of their arithmetic over fields in [32]. In particular, they identified prehomogeneous spaces whose orbits over $\mathbb{Q}$ gave rise to number fields of degrees 4 and 5, but they did not realize their goal of using these to count the fields.

Bhargava has gone through the list of prehomogeneous vector spaces to discover what their nondegenerate integral orbits correspond to, often with unexpected answers. For instance, whereas Wright and Yukie found a surjection taking nondegenerate $\mathrm{GL}_{2}(\mathbb{Q}) \times \mathrm{GL}_{3}(\mathbb{Q})$-orbits in $\mathbb{Q}^{2} \otimes \operatorname{Sym}^{3} \mathbb{Q}^{2}$ to degree 4 number fields, Bhargava discovered that nondegenerate $\mathrm{GL}_{2}(\mathbb{Z}) \times \mathrm{GL}_{3}(\mathbb{Z})$ orbits in $\mathbb{Z}^{2} \otimes \operatorname{Sym}^{3} \mathbb{Z}^{2}$ correspond to commutative rings of rank 4 over $\mathbb{Z}$ equipped with a cubic resolvent ring (a notion he invented). There seems to be no recipe for predicting what extra arithmetic structure is rendered visible by considering integral orbits, but Bhargava has heuristics that have guided him to an answer in each case.

The extra structure can be a nuisance or a boon. It is a nuisance when counting degree 4 fields, for instance, because each field contains many orders (subrings that are free over $\mathbb{Z}$ of the same rank as the field), and each order can have many cubic resolvent rings. But it is a boon if the extra structure itself is worth counting; for instance, one of the prehomogeneous spaces enabled Bhargava [2, Theorem 5] to prove the first nontrivial case of the Cohen-Martinet generalization [18] of the Cohen-Lenstra heuristics on the distribution of class groups.

In Step 2, heuristically the number of lattice points inside a fundamental domain $\mathcal{F}$ should be approximately the volume. If $\mathcal{F}$ has narrowing tentacles stretching to infinity, however, this heuristic can be hard to justify or even false! Bhargava's brilliant solution was to average the counts over several fundamental domains (in fact, to integrate over a continuum of fundamental domains) [2], [4]. This effectively fattens the tentacles so that the volume heuristic can be justified farther out along the tentacle, out to the point beyond which the number of relevant lattice points is provably negligible.

Step 3 is necessary to sieve out unwanted objects. For instance, each number field $k$ has a unique maximal order $\mathcal{O}_{k}$, but the methods in Steps 1 and 2 lead more naturally to a count of all orders, so one must sieve out for each prime $p$ the orders that fail to be maximal at $p$. Ekedahl [22] proved a general sieve result: for instance, given relatively prime $f, g \in \mathbb{Z}\left[x_{1}, \ldots, x_{n}\right]$, he computed the density of what remains of $\mathbb{Z}^{n}$ after sieving out for each $p$ the $\vec{a} \in \mathbb{Z}^{n}$ satisfying $f(\vec{a}) \equiv g(\vec{a}) \equiv 0(\bmod p)$. But Bhargava's applications require sieving out also $\vec{a}$ satisfying congruences of the form $h(\vec{a}) \equiv 0$ $\left(\bmod p^{2}\right)$ for a certain polynomial $h$, and the existing results for the general problem of this kind rely on the $a b c$ conjecture [23], [24]. Bhargava circumvented this for the $h$ arising in his application in a counterintuitive way: he mapped his problem to an Ekedahl-style counting problem in a higherdimensional space. See [6] for the state of the art on this idea.

Ultimately, all these ideas enabled Bhargava to prove that for each $n \leq 5$, the number of isomorphism classes of degree $n$ number fields $k$ satisfying $\left|D_{k}\right| \leq X$ is asymptotic to $c_{n} X$ as $X \rightarrow \infty$ for an explicit constant $c_{n}$ [2], [4]. He was also led to formulate a precise conjecture for higher $n$.

\section{Elliptic Curves}

If $E$ is an elliptic curve $y^{2}=x^{3}+A x+B$ over $\mathbb{Q}$, the set $E(\mathbb{Q})$ of rational points forms an abelian group that is finitely generated. The torsion subgroup of $E(\mathbb{Q})$ is well understood, but the rank as a function of $(A, B)$ is so complicated that it is not even known whether it is computable in theory. Essentially the only known way to bound the rank is to observe that for any $n \geq 2$, the quotient 
$E(\mathbb{Q}) / n E(\mathbb{Q})$ injects into a finite computable group $\operatorname{Sel}_{n} E$ the $n$-Selmer group.

Birch and Swinnerton-Dyer showed that elliptic curves $E$ equipped with a nonzero element of $\operatorname{Sel}_{2} E$ are in bijection with nondegenerate $\mathbb{Q}^{\times} \times \operatorname{PGL}_{2}(\mathbb{Q})$ orbits in $\operatorname{Sym}^{4} \mathbb{Q}^{2}$ and proved that each orbit has an (almost) integral representative [15]. This representation is not a prehomogeneous space, but at least it is coregular, meaning that its ring of polynomial invariants is isomorphic to a polynomial ring. Using knowledge of the invariants, Bhargava and Shankar [10] succeeded in adapting the three-step approach to prove that the average size of $\operatorname{Sel}_{2} E$ as $E$ varies is 3 and hence the average rank of $E(\mathbb{Q})$ is bounded! (See [25] for a summary of their proof.) Although de Jong [20] had proved analogous results over function fields by a similar approach of counting integral orbits and although Brumer [17] had proved that conjectures about elliptic curve $L$-functions would imply that the average rank over $\mathbb{Q}$ was bounded, Bhargava and Shankar were the first to prove unconditionally that the average rank over $\mathbb{Q}$ was bounded and also the first to obtain a precise value for the average of a Selmer group size over a global field.

In subsequent work, Bhargava and Shankar counted integral orbits in more complicated representations to show that for each $n \leq 5$, the average of $\# \operatorname{Sel}_{n} E$ equals the sum of the divisors of $n$. They proved also that the average remains unchanged if finitely many congruence conditions are imposed upon $A$ and $B$. This, combined with a theorem of Dokchitser and Dokchitser [21] relating Selmer groups to root numbers and Wong's method [31, §9] for constructing a positive-density family of elliptic curves in which the root number is equidistributed, implies that the average rank of $E(\mathbb{Q})$ is at most 0.885 [12, Theorem 3]. (Conjecturally the average is $1 / 2$.)

Recent advances in the arithmetic of elliptic curves allow results on Selmer groups of an elliptic curve over $\mathbb{Q}$ to be transferred to results about the rank and analytic rank (the order of vanishing at $s=1$ of the $L$-function of $E$ ). Specifically, in addition to the Dokchitsers' work mentioned above, there is work on the Iwasawa main conjecture for $\mathrm{GL}_{2}$ by Skinner and Urban [28] and by Wan [29]; work connecting $p$-adic $L$-functions to Heegner point heights by Bertolini, Darmon, and Prasanna [1] and by Brooks [16]; and work generalizing the GrossZagier formula by Yuan, Zhang, and Zhang [33]. Combining all of this with the work of Bhargava and Shankar, we now know that

- a positive fraction of elliptic curves over $\mathbb{Q}$ have rank 0 and analytic rank 0 [11] and

- a positive fraction of elliptic curves over $\mathbb{Q}$ have rank 1 and analytic rank 1 [13].

In particular, the Birch and Swinnerton-Dyer conjecture (that rank equals analytic rank) holds for a positive fraction of elliptic curves over $\mathbb{Q}$-in fact, more than 66 percent of them [14].

\section{Higher-Genus Curves}

Bhargava and Gross [7], using work of Wang [30], also found a coregular representation whose rational orbits parametrize 2-Selmer elements of Jacobians of hyperelliptic curves $y^{2}=f(x)$, where $f$ is a polynomial of degree $2 g+1$ over $\mathbb{Q}$ for some fixed $g$. They used this to prove that the average size of the 2-Selmer group of such Jacobians is 3, generalizing the result for elliptic curves. Combining this with Chabauty's $p$-adic method, they could prove that many odd degree hyperelliptic curves have few rational points. Later, Stoll and the present author [26] were able to combine the Bhargava-Gross results with new ideas on the 2-adic geometry of curves to show that as $g \rightarrow \infty$, the fraction of these curves having no rational points at all (other than the one at infinity) tends to 1 .

Bhargava also found a representation providing information on even degree hyperelliptic curves, enabling him to show that for all $g \geq 0$, a positive fraction of curves $y^{2}=f(x)$ with $\operatorname{deg} f=2 g+2$ has no rational points (he showed that their 2coverings fail to have local points) and the fraction tends to 1 as $g \rightarrow \infty$ [5]. Later, with Gross and Wang he showed that a positive fraction of these curves also has no points over any number field of odd degree despite having points over every completion of $\mathbb{Q}[8]$.

Combining the results for odd and even degrees, we now know that most equations of the form $y^{2}=f(x)$ have no rational solutions.

\section{Final Words}

There are many other beautiful works of Bhargava we did not have space to discuss, such as a vast generalization [3] of Mahler's theorem on series expansions for $p$-adic continuous functions and a preprint with Hanke [9] on Conway's "290conjecture" that a positive-definite quadratic form over $\mathbb{Z}$ represents all nonnegative integers if and only if it represents all nonnegative integers up to 290. But it is the counting techniques that have had the greatest impact. Bhargava's ideas will surely exert a strong influence on the future development of the subject.

\section{Acknowledgments}

I thank Manjul Bhargava, Tim Dokchitser, Andrew Granville, and Benedict Gross for comments.

\section{References}

[1] MASSIMO Bertolini, HenRI DARMON, and KARTIK PRASANNA, with an appendix by Brian Conrad, Generalized Heegner cycles and $p$-adic Rankin $L$-series, Duke Math. J. 162 (2013), no. 6, 1033-1148, DOI 10.1215/00127094-2142056. MR3053566 
[2] Manjul Bhargava, The density of discriminants of quartic rings and fields, Ann. of Math. (2) 162 (2005), no. 2, 1031-1063. MR2183288

[3] _ , On $P$-orderings, rings of integer-valued polynomials, and ultrametric analysis, J. Amer. Math. Soc. 22 (2009), no. 4, 963-993, DOI 10.1090/S0894-0347-09-006389. MR2525777 (2010i:13022)

[4] _ The density of discriminants of quintic rings and fields, Ann. of Math. (2) 172 (2010), no. 3, 15591591, DOI 10.4007/annals.2010.172.1559. MR2745272 (2011k:11152)

[5] , Most hyperelliptic curves over $\mathbb{Q}$ have no rational points, August 5, 2013. Preprint, arXiv:1308.0395v1 [6] _ , The geometric sieve and the density of squarefree values of invariant polynomials, January 31, 2014. Preprint, arXiv:1402.0031v1.

[7] Manjul BhaRgaVA and Benedict H. GRoss, The average size of the 2-Selmer group of Jacobians of hyperelliptic curves having a rational Weierstrass point, Automorphic representations and L-functions, Tata Inst. Fundam. Res. Stud. Math., vol. 22, Tata Inst. Fund. Res., Mumbai, 2013, pp. 23-91. MR3156850

[8] Manjul BHARGAVA, BENEDICT H. GROSS, and XiAOHENG WANG, with an appendix by Tim and Vladimir Dokchitser, Pencils of quadrics and the arithmetic of hyperelliptic curves, October 29, 2013. Preprint, arXiv:1310.7692v1, to appear in J. Amer. Math. Soc.

[9] MANJUl Bhargava and JONATHAN HANKE, Universal quadratic forms and the 290-theorem, November 28, 2011. Preprint, http://www.wordpress. jonhanke.com/ ?page_id=363.

[10] MANJUl BHARGAVA and ARUL SHANKAR, Binary quartic forms having bounded invariants, and the boundedness of the average rank of elliptic curves, December 23, 2013. Preprint, arXiv:1006.1002v3, to appear in Annals of Math.

[11] _ Ternary cubic forms having bounded invariants, and the existence of a positive proportion of elliptic curves having rank 0, December 24, 2013. Preprint, arXiv:1007.0052v2.

[12] elliptic curves is 6 , and the average rank is less than 1 , December 30, 2013. Preprint, arXiv:1312.7859v1.

[13] MANJUl BHARGAVA and CHRISTOPHER SKINNER, A positive proportion of elliptic curves over $\mathbb{Q}$ have rank one, J. Ramanujan Math. Soc. 29 (2014), no. 2, 221-242. MR3237733

[14] MANJul BHARgava, CHRISTOPHER SKINNER, and WeI ZHANG, A majority of elliptic curves over $\mathbb{Q}$ satisfy the Birch and Swinnerton-Dyer conjecture, July 17, 2014. Preprint, arXiv: 1407.1826v2

[15] B. J. BirCH and H. P. F. SWINNERTON-Dyer, Notes on elliptic curves. I, J. Reine Angew. Math. 212 (1963), 7-25. MR0146143 (26 \#3669)

[16] ERnEST HunTER BRoOKS, Generalized Heegner cycles, Shimura curves, and special values of $p$-adic L-functions, 2013. PhD thesis, Univ. of Michigan. Available at http://dept.math.1sa.umich.edu/research/ number_theory/theses/hunter_brooks.pdf.

[17] ARMAND BRUMER, The average rank of elliptic curves. I, Invent. Math. 109 (1992), no. 3, 445-472, DOI 10.1007/BF01232033. MR1176198 (93g:11057)

[18] HenRI COHEN and JACQUES MARTINET, Etude heuristique des groupes de classes des corps de nombres, J. Reine Angew. Math. 404 (1990), 39-76 (French). MR1037430 (91k:11097)
[19] H. DAVEnport and H. Heilbronn, On the density of discriminants of cubic fields. II, Proc. Roy. Soc. London Ser. A 322 (1971), no. 1551, 405-420. MR0491593 (58 \#10816) [20] A. J. DE JonG, Counting elliptic surfaces over finite fields, Mosc. Math. J. 2 (2002), no. 2, 281-311. Dedicated to Yuri I. Manin on the occasion of his 65 th birthday. MR1944508 (2003m:11080)

[21] TIM DOKCHITSER and Vladimir DOKChITSER, On the Birch-Swinnerton-Dyer quotients modulo squares, Ann. of Math. (2) 172 (2010), no. 1, 567-596, DOI 10.4007/annals.2010.172.567. MR2680426 (2011h:11069) [22] TORSTEN EKEDAHL, An infinite version of the Chinese remainder theorem, Comment. Math. Univ. St. Paul. 40 (1991), no. 1, 53-59. MR1104780 (92h:11027)

[23] ANDREW GRANVILLE, $A B C$ allows us to count squarefrees, Internat. Math. Res. Notices 19 (1998), 991-1009. MR1654759 (99j:11104)

[24] BJORN POONEN, Squarefree values of multivariable polynomials, Duke Math. J. 118 (2003), no. 2, 353-373. MR1980998 (2004d:11094)

[25] _ Average rank of elliptic curves [after Manjul Bhargava and Arul Shankar], Séminaire Bourbaki. Vol. 2011/2012. Exposés 1043-1058, Astérisque 352 (2013), Exp. No. 1049, viii, 187-204. MR3087347

[26] BJORn POONEN and Michael STOLL, Most odd degree hyperelliptic curves have only one rational point, Ann. of Math. (2) 180 (2014), no. 3, 1137-1166.

[27] M. SATO and T. KIMURA, A classification of irreducible prehomogeneous vector spaces and their relative invariants, Nagoya Math. J. 65 (1977), 1-155. MR0430336 (55 \#3341)

[28] Christopher SKInNer and ERIC Urban, The Iwasawa main conjectures for GL2, November 2010. Preprint, www. math.columbia.edu/ urban/eurp/MC.pdf.

[29] XIN WAN, Iwasawa Main Conjecture for RankinSelberg $p$-adic $L$-functions, August 18, 2014. Preprint, arXiv: 1408.4044v1.

[30] XIAOHENG JERRY WANG, Pencils of quadrics and Jacobians of hyperelliptic curves, 2013. $\mathrm{PhD}$ thesis, Harvard University. Available at nrs.harvard.edu/urn-3:HUL. InstRepos: 11156784

[31] SIMAN WONG, On the density of elliptic curves, Compositio Math. 127 (2001), no. 1, 23-54, DOI 10.1023/A:1017514507447. MR1832985 (2002d:11066) [32] DAVID J. Wright and AKIHIKO YuKIE, Prehomogeneous vector spaces and field extensions, Invent. Math. 110 (1992), no. 2, 283-314, DOI 10.1007/BF01231334. MR1185585 (93j:12004)

[33] Xinyi Yuan, Shou-Wu Zhang, and Wei Zhang, The Gross-Zagier Formula on Shimura Curves, Annals of Mathematics Studies, vol. 184, Princeton University Press, Princeton, NJ, 2013. MR3237437 


\section{Jeremy Quastel}

\section{The Work of Martin Hairer}

Martin Hairer of the University of Warwick was awarded a Fields Medal at the 2014 ICM in Seoul for his outstanding contributions to the theory of stochastic partial differential equations (SPDE). His most spectacular achievement has been his single-handed creation of the theory of regularity structures, which is a flexible analytic tool for giving sense to many ill-posed SPDEs. This breakthrough has opened up the entire field. Hairer is an Austrian who was raised in a mathematical family in Geneva, where he obtained a PhD in physics under JeanPierre Eckmann. But those who understand his work know that he is a mathematician in the best sense of the word: a quick study, patient, genial, and well rounded. His work ranges over many aspects of physically motivated problems in (stochastic) analysis. Here I will concentrate on explaining a few of his works in SPDE. First, some background.

\section{Markov Processes}

Markov processes are used widely in the sciences to model dynamical processes whose evolution is in some way random. They are defined through transition probabilities $p(t, x, A)$ representing the probability for the state $X_{t+s}$ at future time $t+s$ to be in a set $A$, given that at the present time $s$, $X_{s}=x$. If we had explicit transition probabilities and an initial measure $\mu_{0}$ in hand, we could in principle compute everything. However, they are usually presented to us only implicitly, the state evolving according to some sort of stochastic equation. Even in the case of stochastic ordinary differential equations, $X_{t} \in \mathbb{R}^{d}, t>0$,

$$
\dot{X}_{t}=b\left(X_{t}\right)+\sigma\left(X_{t}\right) \xi_{t}
$$

where in the natural simplest cases $\xi_{t}$ is Gaussian white noise, it is not obvious how to make sense of the equation. White noise means that averages of $\xi_{t}$ over nice disjoint sets are uncorrelated, or, in other words, $B_{t}=\int_{0}^{t} \xi_{s} d s$ is Brownian motion. One of the first things one learns is that with probability 1 it is nowhere differentiable; in fact, it just fails to be Hölder $1 / 2$. From the equation, we expect the same of $X_{t}$. A classical fact is that the Riemann-Stieltjes integral $\int F d G$ is ill defined if the Hölder exponents of $F$ and $G$ sum to less than 1. Hence one cannot give unambiguous meaning for typical realizations of the noise to the stochastic integral which is the last term in the integrated version of (1),

$$
X_{t}=x+\int_{0}^{t} b\left(X_{s}\right) d s+\int_{0}^{t} \sigma\left(X_{s}\right) d B_{s} .
$$

Jeremy Quastel is professor of mathematics at the University of Toronto. His email address is quaste1@math. toronto.edu.
However, the difference between various approximations turns out to have a probabilistically well-defined limit, and therefore the ambiguity can be understood as a modelling issue. This was worked out by Itô and others, the key concept being martingales. A downside is lack of continuity: If $\xi_{t}^{\varepsilon}$ are smooth approximations to the white noise, $\int_{0}^{t} \sigma\left(X_{S}^{\varepsilon}\right) \xi_{s}^{\varepsilon} d s$ may not even converge to $\int_{0}^{t} \sigma\left(X_{s}\right) \xi_{s} d s$. A modern point of view [8] due to Terry Lyons clarifies the approximation issue by noting that once one has made a choice of the simplest ambiguous quantity $\mathbb{B}_{t}:=\int_{0}^{t} B_{S} d B_{S}$, the rest is ordained. In particular, the solution map $B_{t} \mapsto X_{t}$ factors into a probabilistic step in which one enhances the Brownian motion $B_{t}$ with an admissible choice of $\mathbb{B}_{t}$, together with an explicit, deterministic, continuous map from the resulting rough path $\left(B_{t}, \mathbb{B}_{t}\right)$ to the solution $X_{t}$.

Another important question about Markov processes concerns long time behavior. A nice situation is when the process is ergodic-there exists a unique invariant measure-and after a long time basically finds itself in the temporally stationary process started with the invariant measure.

In the finite-dimensional setting of (1), these problems can be studied through the operators

$$
L=\frac{1}{2} \sum_{i, j=1}^{d} a_{i j}(x) \frac{\partial^{2}}{\partial x_{i} \partial x_{j}}+\sum_{i=1}^{d} b_{i}(x) \frac{\partial}{\partial x_{i}}
$$

where $a=\sigma \sigma^{*}$. The transition probabilities are the kernels of the semigroup $e^{t L}$ and invariant measure solutions of $L^{*} \mu=0$. If the variance matrix $a$ is degenerate, the uniqueness follows from the Hörmander hypoellipticity theorem, probabilistic proofs of which can be obtained using the stochastic calculus of variations a.k.a. Malliavin calculus.

\section{SPDE}

However, many physically motivated problems are presented to us as stochastic partial differential equations, in particular, several equations for processes which arise as canonical representatives of huge fluctuation universality classes. Despite several decades of work, methods which could deal with these important examples proved elusive, and a general theory was not expected. Now $d$ in (3) is replaced by a continuum $\mathbb{R}^{d}$, and there exists no useful infinite-dimensional PDE theory to help us. Many of these equations are far more ill-posed than (1), where we were just marginally below the regularity at which things would have made classical sense. These singular SPDEs are relations between nonlinear functions of various derivatives of nowhere differentiable functions which are not even close to making classical sense. They seemed to contain some magical hidden meaning which could not be made precise. 


\section{Ergodicity of Navier-Stokes}

An SPDE problem where the long time behavior is of obvious interest (e.g. in turbulence) is that of a randomly forced fluid. We have the incompressible Navier-Stokes equations which ask for a divergencefree vector field evolving according to

$$
\frac{\partial u}{\partial t}+(u \cdot \nabla) u+\nabla P=v \Delta u+F .
$$

$P$ is the pressure, and $F$ is the external force. We assume $F$ is acting in just a few long wavelength modes (i.e. someone is stirring it). Physically, the injected energy cascades down to smaller scales in the inertial range until it gets to a smallest scale, at which it is dissipated by the viscosity $v$, which should be thought of as small. A natural question then is whether there is a unique invariant measure supported in this inertial range. One works in two dimensions (in $d=3$ simply making sense of the unforced equation for more than a short time is already a millennium problem). Since the forcing is in only a few modes, the problem is highly hypoelliptic and becomes that of obtaining results that compare favorably to the Hörmander condition, but now in infinite dimensions. A key difficulty is lack of natural reference measuresit is just too easy to be singular in infinite dimensions - and a subsequent lack of good norms for the dynamics. The problem attracted the interest of several groups in the early 2000s [7], [17], [3]; with the finite-dimensional forcing it was finally solved by Hairer and Mattingly [10] with a condition allowing a forcing on only two Fourier modes for any $v>0$ ! An asymptotic Feller property is introduced which is sensitive to the regularization of the transition densities due to both probabilistic and dynamic mechanisms, and this in turn is verified through the nondegeneracy of the Malliavin covariance matrix. Naturally there are extremely interesting and important questions, such as the distribution of energy in these invariant measures, for which there are physical predictions which await mathematically rigorous work.

\section{The KPZ Revolutions}

In the mid-1980s, Kardar, Parisi and Zhang introduced the equation

$$
\partial_{t} h=\lambda\left(\partial_{x} h\right)^{2}+\partial_{x}^{2} h+\xi
$$

to describe a randomly growing interface in $d=2$, such as the boundary of a bacterial colony or a slowly burning front, idealized to be a height function $h(t, x), t>0, x \in \mathbb{R}$. The right-hand side identifies the three key mechanisms of relaxation $\left(\partial_{x}^{2} h\right)$, uncorrelated random forcing (space-time white noise $\xi$ ), and, most important, the nonlinear slope dependent drift or lateral growth $\left(\partial_{x} h\right)^{2}$.

If the asymmetry $\lambda$ is set to 0 , the equation becomes linear (in the noise) and readily integrated, yielding a continuous function-valued Markov process which has Brownian motion as invariant measure. A smooth initial function evolves for $t>0$ to a locally Brownian version and in the long time limit after centering, to Brownian motion.

Amazingly, the nonlinear dynamics also preserves Brownian motion (modulo an absolute height shift), but the fluctuations are otherwise non-Gaussian and of nonstandard superdiffusive size $t^{1 / 3}$ (compared to $t^{1 / 4}$ when $\lambda=0$ ). At the physical level, this goes back to highly nonrigorous renormalization group computations on the stochastic Burgers equation satisfied by $u=\partial_{x} h$.

In a breakthrough that surprised both the mathematics and physics communities, Johannson [15] (and [1] for a related model) succeeded in computing the free energy fluctuations of geometric last passage percolation and showing that normalized by $t^{1 / 3}$ they converge to the TracyWidom law, which had arisen ten years earlier in the seemingly unrelated field of random matrices. These free energies satisfy discrete versions of the $\mathrm{KPZ}$ equation, and at the physical level one expects all such one-dimensional systems displaying the three basic mechanisms to have the same large time asymptotics, refined into asymptotic fluctuation classes depending only on initial data. This is the strong KPZ universality conjecture, and the poorly understood universal large time limit is known as the KPZ fixed point. There has been significant progress over the last fifteen years through the work of Spohn, Sasamoto, Tracy, Widom, le Doussal, Dotsenko, Borodin, Corwin, O'Connell, Seppäläinen, Ferrari, and others on a small number of exactly solvable models, including to some extent the KPZ equation itself, where a few exact one-point distributions have been computed. However, at this level KPZ is just one model within the huge universality class. The connection with random matrices and the origin of the exact solvability and its link to representation theory have been considerably clarified by the work of Borodin and coauthors, especially under the umbrella of Macdonald Processes [2].

However, the KPZ equation is also itself universal in the sense that it is expected to be the unique heteroclinic orbit connecting (by varying time $t$ or equivalently $\lambda$ ) the Gaussian fixed point to the KPZ fixed point and consequently the universal fixed point of models with weakly tuned asymmetry or noise. This is the weak KPZ universality conjecture. In contrast to the strong conjecture, where at the present time we can rely only on fortuitous exact computations coupled with asymptotics, the weak conjecture is open to a general attack by mathematical analysis.

To attempt it, one needs an analytic theory of KPZ. But if $h(t, x)$ in (5) is locally Brownian in $x$, what could the nonlinear term $\left(\partial_{x} h\right)^{2}$ possibly mean? Of course, the problem of finding ways to multiply genuine Schwartz distributions is an old 
one, and various idiosyncratic techniques existed, but here one has to find the definition imposed by the physics. It was far from clear how to accomplish this, and earlier attempts turned out not to be physical [4].

Actually, for the KPZ equation, there is a cheap way out. At a completely formal level,

$$
Z(t, x)=\exp h(t, x)
$$

satisfies

$$
\partial_{t} Z=\partial_{x}^{2} Z+\xi Z
$$

Due to the roughness of $\xi$, there is really an infinite absolute height shift. But we are just looking at fluctuations, so a huge change of reference frame doesn't matter. The multiplicative stochastic heat equation (7) was one of the few truly infinite-dimensional nonlinear SPDEs which could be handled by the Itô techniques. The resulting $h:=\log Z$ is known as the Hopf-Cole solution of KPZ, and it is now known to have the expected physical behavior arise in the weakly asymmetric/weak noise limits and is what the exact computations refer to, the remarkable fact being that the correlations, i.e. the noise averages $E\left[Z\left(t, x_{1}\right) \cdots Z\left(t, x_{n}\right)\right]$, satisfy closed Bethe ansatz solvable delta-Bose gas equations.

This suggests that one way to prove the weak universality conjecture would be to show that the appropriate partition functions converge to (7). This was done first by Bertini and Giacomin for a model called asymmetric simple exclusion and then later for a few others. But only very special models can be handled in this manner; one is faced again with the problem that the Itô theory is not well set up for approximations, and it just begs the question of finding a more robust theory for nonlinear SPDE.

\section{Regularity Structures}

A natural approach to make sense of a singular SPDE is through regularization. For example, we could take smooth approximations $\xi_{\varepsilon}$ to our noise $\xi$. If we can somehow find a limit of the resulting solutions as the regularization is removed that doesn't depend on the way we regularize, we can say it is the solution. But typically the solution map lacks continuity in the necessary topology, and convergence is false. However, it may converge after renormalization by some probabilistically well-defined but now divergent quantity. For KPZ

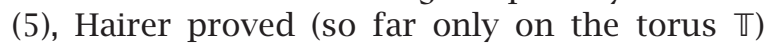
that there is a diverging $C_{\varepsilon}$ such that the solution $h_{\varepsilon}(t, x)$ of

$$
\partial_{t} h_{\varepsilon}=\lambda\left[\left(\partial_{x} h_{\varepsilon}\right)^{2}-C_{\varepsilon}\right]+\partial_{X}^{2} h_{\varepsilon}+\xi_{\varepsilon}
$$

converges to the Hopf-Cole solution, in probability, locally uniformly as continuous functions. If the regularization of the noise were just in space, this could be done using the Hopf-Cole + Itô approach, but the key point is that the new method is general and robust. To give just one example, if the regularization of the noise is on a scale $\varepsilon$ in space and $\varepsilon^{2}$ in time, the weak KPZ universality suggests that the classical solution to

$$
\partial_{t} \hat{h}_{\varepsilon}=\varepsilon\left(\partial_{x} \hat{h}_{\varepsilon}\right)^{4}-C_{\varepsilon}^{\prime}+\partial_{x}^{2} \hat{h}_{\varepsilon}+\xi_{\varepsilon}
$$

should converge to the Hopf-Cole solution of (5) with a highly nontrivial $\lambda$, the exponent 4 miraculously becoming a 2 in the limit. Hairer's method can be adapted to prove such things (see [13] for a review) as well as providing a good approximation theory for equations like (7) [14]. The interested reader is invited to write the equation for $\hat{Z}=\log \hat{h}$ and try to understand why on Earth it would converge to (7).

Motivated by rough paths, the idea is to factor the solution map into a deterministic part and a probabilistic part. The deterministic part is provided by an abstract solution map which acts on an abstract version of the equation together with a lift of the noise. The probabilistic part, which has some degrees of freedom, is how you lift the noise to the abstract framework.

The main new tool to construct the abstract solution map is the notion of regularity structures. This is a vector space tailor-made for the equation, with sufficient information to provide a local description of the solution at each point at various levels of regularity. It contains polynomials in the time and space variables, like a classical Taylor expansion. But our solution is going to be built out of various functionals of the noise which are too rough to represent using only these. So it should also contain symbols representing the input noise and enough rules for multiplication and versions of the heat operator and differentiation so that we can represent our equation there. One also needs a group of transformations from the description at one point to another point, and it is here that a highly nontrivial algebraic structure arises. Next one builds a space of functions $\mathcal{D}$ taking values in the regularity structure that generalizes the classical Hölder spaces and on which there are analogues of the Schauder estimates for the heat operator. These allow one to obtain the solution there as a fixed point.

Finally, a reconstruction operator pastes together the expansions at different points so that the abstract solution is realized as a genuine Schwartz distribution, which (one hopes!) is the solution to the original equation.

It is indeed the solution for the naïve lift of the regularized noise. But as we remove the regularization, things do not converge. Fortunately, a renormalization group acts on the space of lifts of the noise/multiplication rules, and by a careful choice one can find a sequence which does converge as one removes the regularization. These renormalizations are realized as concrete 
renormalizations of the equations satisfied by the reconstruction; in the case of KPZ we get (8), and the Hopf-Cole limit can be thought of as at best satisfying

$$
\partial_{t} h=\lambda\left[\left(\partial_{x} h\right)^{2}-\infty\right]+\partial_{x}^{2} h+\xi .
$$

In this way, the problem is reduced to finding the correct renormalization, usually by educated guesswork, and then proving convergence of the corresponding finite collection of multilinear transformations of the noise.

If the smooth approximating noises are Gaussian, we are in a situation of Wiener chaoses, and there are well-known criteria for convergence based on $L^{2}$ norms. While these are completely explicit, they are generally complicated convolutions of singular kernels, and convergence depends on a careful counting of singularities in integrals over a resulting graph. This is highly reminiscent of earlier calculations in quantum field theory, and Feynman-like diagrams are used to keep track of the cumbersome computations.

\section{Dynamic $\Phi_{3}^{4}$}

The $\Phi_{d}^{4}$ model is supposed to be the Gibbs probability measure on Schwartz distributions $\Phi$ on $\mathbb{R}^{d}$, or the torus $\mathbb{T}^{d}$ or some subset, where the probability of $\Phi$ is proportional to $e^{-\beta H(\Phi)}$ where $H(\Phi)=\int\left[|\nabla \Phi|^{2}+\frac{C}{2}|\Phi|^{2}-\frac{\lambda}{4} \Phi^{4}\right]$. It is a universal model for phase coexistence in near-critical phenomena, closely related to SPDE and similarly ill-posed in $d \geq 2$, since the regularity of the Gaussian field obtained by setting $\lambda=0$ is insufficient to define $\int \Phi^{4}$. So we take some regularization and try to find a nontrivial (i.e. non-Gaussian) limit as it is removed, perhaps after some renormalization. The problem was well studied, the main result being that it is only nontrivial in $d \leq 3$. An outstanding issue was whether the limits resulting from different regularizations in the difficult $d=3$ case were really the same. Similar to the SPDE problems, what was missing was an intrinsic characterization of the limit.

It was suggested that by analogy with the Ising model, where information about the static model had been obtained from the Glauber dynamics, one might approach this through a study of the stochastic dynamics ("stochastic quantization") with $\Phi_{3}^{4}$ as invariant measure:

$$
\partial_{t} \Phi=\Delta \Phi+\lambda \Phi^{3}-C \Phi+\xi .
$$

It is expected to be a universal model for such dynamical models near criticality in a limit similar to the weak limits for KPZ.

But (11) just opened a new can of worms; for twenty years nobody could even make sense of it in $d=3$ until Hairer constructed the appropriate regularity structure, in which the solution exists as an abstract model to which lifts of appropriate renormalized solutions of regularized versions of (11) converge.

Since Hairer's first well-posedness results, several proofs have appeared for KPZ [9] and dynamic $\Phi_{3}^{4}$ [16], [5]. However these appear to be far more closely tied to the particular regularization as well as the specifics of the equation. One of the key points of the regularity structures is that they do give an intrinsic characterization of solutions for a large class of equations like (5) or (11) as the solution in the appropriate $\mathcal{D}$ with the lift of the rough noise. The condition is that the equation should be subcritical, meaning that as you zoom in, the nonlinearity vanishes. In addition, regularity structures provide a precise description of the solution, level by level of (ir)regularity.

\section{References}

[1] J. BAIK, P. A. DEIFT, and K. JohAnsson, On the distribution of the length of the longest increasing subsequence of random permutations, J. Amer. Math. Soc. 12 (1999), 1119-1178.

[2] A. Borodin and I. CoRwIn, Macdonald processes, Probab. Th. Rel. Fields 158 (2014), no. 1-2, 225-400.

[3] J. BRICMONT, A. KUPIAINEN, and R. LEFEVERE, Ergodicity of the 2D Navier-Stokes equations with random forcing, Comm. Math. Phys. 224 (2001), 65-81.

[4] T. CHAN, Scaling limits of Wick ordered KPZ equation, Comm. Math. Phys. 209 (2000), 671-690.

[5] R. CATEllier and K. ChOuK, Paracontrolled distributions and the 3-dimensional stochastic quantization equation, arXiv:1310.6869

[6] I. CoRwIN, The Kardar-Parisi-Zhang equation and universality class, Random Matrices Theory Appl. 1 (2012), no. 1.

[7] W. E. J. C. MAtTinglY and Y. G. SinAI, Gibbsian dynamics and ergodicity for the stochastic forced Navier-Stokes equation, Comm. Math. Phys. 224 (2001), 83-106.

[8] P. Friz AND M. HAIRER, A Course on Rough Paths, Springer, 2014.

[9] M. Gubinelli and N. PeRKowsKi, KPZ reloaded, arXiv:1508.03877

[10] M. HAIRER and J. C. MATTINGLY, Ergodicity of the 2D Navier-Stokes equations with degenerate stochastic forcing, Ann. of Math. (2) 164 (2006), no. 3, 993-1032.

[11] M. HAIRER, Solving the KPZ equation, Ann. of Math. (2) 178 (2013), no. 2, 559-664.

[12] M. HAIRER, A theory of regularity structures, Invent. Math. 198 (2014), no. 2, 269-504.

[13] M. HAIRER, Regularity structures and the dynamical $\Phi_{3}^{4}$ model, arXiv:1508.05261

[14] M. HAIRER and E. PARDOUX, A Wong-Zakai theorem for stochastic PDEs, arXiv:1409.3138

[15] K. JOHANSSON, Shape fluctuations and random matrices, Comm. Math. Phys. 209 (2000), 437-476.

[16] A. KUPIAINEN, Renormalization group and stochastic PDEs, arXiv:1410.3094

[17] S. KuKSIN and A. SHIRIKYAN, Stochastic dissipative PDEs and Gibbs measures, Comm. Math. Phys. 213 (2000), 291-330. 


\section{Anton Zorich}

\section{The Work of Maryam Mirzakhani}

On August 13, 2014 (the opening day of ICM at Seoul), Maryam Mirzakhani received the Fields Medal "for her outstanding contributions to the dynamics and geometry of Riemann surfaces and their moduli spaces," becoming the first woman to win the Fields Medal. We outline several directions of Mirzakhani's research, which ranges from geometry of hyperbolic surfaces to dynamics in moduli spaces passing through applications of symplectic topology to algebraic geometry of moduli spaces. More scientific details can be found in the presentation of $\mathrm{C}$. McMullen for the ICM [McM] and in a very accessible article [W] of A. Wright. For a more personal biographical note we recommend the paper of E. Klarreich [Kl].

\section{Moduli Spaces}

We are used to the fact that geometric objects might form continuous families with a rich topology. For example, the family of all straight lines in the plane passing through the origin forms a circle; the family of all complex lines passing through the origin of the vector space $\mathbb{C}^{n+1}$ forms the complex projective space $\mathbb{C} \mathrm{P}^{n}$.

One can consider continuous families of certain geometric structures on a fixed manifold. For example, the family of all possible complex structures on a smooth compact surface $S$ of genus $g$ forms the moduli space $\mathcal{M}_{g}$ of complex dimension $3 g-3$. By the uniformization theorem complex structures on a smooth surface are in natural bijection with hyperbolic metrics of constant curvature, so the moduli space $\mathcal{M}_{\mathfrak{g}}$ can also be seen as the family of nonsingular metrics of a fixed negative curvature on the surface $S$.

During the last several decades various moduli spaces became very common in mathematics and theoretical physics. When working on extremely naive objects, such as graphs or interval exchange transformations, one might run into questions related to moduli spaces.

Theoretical physics continually develops its opinion on the nature of the relevant space which we inhabit. Strings give way to d-branes, and moduli spaces of Riemann surfaces give way to moduli spaces of Calabi-Yau manifolds.

While the geometry of the world most of us inhabit is not yet clear, what is clear is that Maryam Mirzakhani has spent many years in the worlds of hyperbolic and flat surfaces.

Anton Zorich is a professor at the Institut de mathématiques de Jussieu-Paris Rive Gauche and a member of the Institut Universitaire de France. His email address is anton.zorich@imj-prg.fr.

\section{The Hyperbolic World}

\section{Weil-Petersson Volumes}

Consider a smooth surface $S$ of genus $g$ with $n$ holes (where $n=0$ is not excluded). A closed curve $\alpha$ on $S$ is called simple if it does not have self-intersections. Speaking about simple closed curves on a surface $S$ we always tacitly assume that they are not contractible either to a point or to one of the boundary components (if there are any).

Suppose now that the surface $S$ is endowed with a metric of constant curvature -1 . By convention, we always assume that the boundary components of the resulting hyperbolic surface $X$ are realized by geodesics $\beta_{i}$ in the hyperbolic metric, where $i=1, \ldots, n$. The hyperbolic lengths of the geodesic boundary components $\beta_{i}$ are denoted by $b_{i}(X)$ or by $L_{i}(X)=\left|\beta_{i}\right|_{X}$. By convention, the zero value $L_{i}=b_{i}=0$ corresponds to a cusp of the hyperbolic metric.

Fixing the hyperbolic lengths $b_{i}$ of all boundary components and varying the hyperbolic metric, we get a continuous family of hyperbolic metrics on $S$. The real $(6 g-6+2 n)$-dimensional space of all such metrics is called the moduli space $\mathcal{M}_{g, n}\left(b_{1}, \ldots, b_{n}\right)$ of bordered hyperbolic surfaces. By the work of W. Goldman and S. Wolpert it carries a natural closed 2 -form $\omega_{W P}$ called the Weil-Petersson symplectic form.

The wedge power $\omega^{n}$ of a symplectic form on a manifold $M^{2 n}$ defines a volume form. The volume of the moduli space $\mathcal{M}_{g, n}\left(b_{1}, \ldots, b_{n}\right)$ with respect to the volume form $\omega_{W P}^{3 g-3+n}$ is called the Weil-Petersson volume of the moduli space $\mathcal{M}_{g, n}\left(b_{1}, \ldots, b_{n}\right)$. To give an account of Mirzakhani's work on Weil-Petersson volumes, we start with the mysterious identity of G. McShane.

Theorem (McShane). Let $f(x)=\left(1+e^{x}\right)^{-1}$ and let $X$ be a hyperbolic torus with a cusp. Then

$$
\sum_{\gamma} f\left(\ell_{\gamma}(X)\right)=\frac{1}{2},
$$

where the sum is taken over all simple closed geodesics $\gamma$ on $X$, and $\ell_{\gamma}(X)$ is the length of the geodesic $\gamma$.

This identity is in some sense a miracle: though the length spectrum of simple closed geodesics is different for different hyperbolic tori with a cusp, the sum above is identically $1 / 2$ for any $X \in \mathcal{M}_{1,1}(0)$. Ten years after the work of McShane, Mirzakhani discovered a remarkable generalization of McShane's identity to hyperbolic surfaces of any genus with any number of boundary components.

Let us discuss why such identities are relevant to the Weil-Petersson volumes of the moduli spaces. Integrating the right-hand side of McShane's identity over the moduli space $\mathcal{M}_{1,1}(0)$ with respect to the Weil-Petersson form, one obviously gets 
$\frac{1}{2} \operatorname{Vol} \mathcal{M}_{1,1}(0)$. What is not tautology is that the integral of the sum on the left-hand side admits a geometric interpretation as the integral of $f$ over the natural cover $\mathcal{M}_{1,1}^{*}(0)$ of the initial moduli space $\mathcal{M}_{1,1}(0)$. This cover is already much simpler than the original moduli space: it admits global coordinates in which the integral of $f$ can be easily computed.

Mirzakhani's more general identity does not immediately yield the volume. However, cutting the initial surface by simple closed geodesics involved in her identity and developing the idea of averaging over all possible hyperbolic surfaces, Mirzakhani gets a recursive relation for the volume $V_{g, n}\left(L_{1}, \ldots, L_{n}\right):=\operatorname{Vol} \mathcal{M}_{g, n}(L)$ in terms of analogous volumes of simpler moduli spaces. These relations allow Mirzakhani to prove the following statement and to compute the volumes explicitly.

Theorem ([M2]). The volume $V_{g, n}\left(L_{1}, \ldots, L_{n}\right)$ is a polynomial in $L_{1}^{2}, \ldots, L_{n}^{2}$; namely, we have

$$
V_{g, n}(L)=\sum_{|\alpha| \leq 3 g-3+n} C_{\alpha} \cdot L^{2 \alpha},
$$

where $C_{\alpha}>0$ lies in $\pi^{6 g-6+2 n-2|\alpha|} \cdot \mathbb{Q}$.

Simple recursive formulae for volumes in genera $0,1,2$ were found earlier by P. Zograf. Very precise asymptotics of volumes for large genera were recently proved by M. Mirzakhani and P. Zograf [MZg] (up to a multiplicative constant conjecturally equal to $\frac{1}{\sqrt{\pi}}$ which still resists a rigorous evaluation).

\section{Counting Simple Closed Geodesics}

Consider a hyperbolic surface $X$ of finite area. In this section there are no boundary components, but we still allow the hyperbolic metric of constant curvature -1 to have cusps. We denote the corresponding moduli space by $\mathcal{M}_{g, n}$. It is known that the growth rate of the number of closed geodesics of length at most $L$ on any such surface $X$ has the rate $e^{L} / L$ when the bound $L$ grows.

It is not surprising that most long closed geodesics have self-intersections. A quantitative estimate of "most" is much more subtle: M. Rees and I. Rivin showed that the number $s_{X}(L)$ of simple closed geodesics of length at most $L$ grows polynomially in $L$.

In counting geodesics, one can count separately those simple closed geodesics which separate $X$ into two connected components and those which do not. More generally, two simple closed curves on a topological surface $S$ have the same topological type if one curve can be transformed to another by a homeomorphism of $S$. For any surface of a fixed genus $g$ with a fixed number $n$ of punctures the number of different topological types of simple closed curves is finite. M. Mirzakhani counted simple closed hyperbolic geodesics type by type.
Theorem ([M3]). For any hyperbolic surface $X \in \mathcal{M}_{g, n}$ the number $s_{X}(L$, type $)$ of simple closed geodesics on $X$ of length at most $L$ and of a fixed topological type has exact polynomial asymptotics:

$$
\lim _{L \rightarrow+\infty} \frac{s_{X}(L, \text { type })}{L^{d}}=c(\text { type }) \cdot \operatorname{Vol} B_{1}^{T h}(X),
$$

where $d=6 g-6+2 n=\operatorname{dim}_{\mathbb{R}} \mathcal{M}_{g, n}$.

For geometers we add that the quantity $\operatorname{Vol} B_{1}^{T h}(X)$ is the normalized volume of the "unit ball" in the space of measured laminations $\mathcal{M} \mathcal{L}_{g, n}$ where the "unit ball" is defined in terms of the hyperbolic metric on $X$ and its volume is computed in terms of the Thurston measure on $\mathcal{M} \mathcal{L}_{g, n}$.

Note that the asymptotic proportion of simple closed geodesics of fixed type is the same for all hyperbolic surfaces $X$ in $\mathcal{M}_{g, n}$ :

$$
\lim _{L \rightarrow+\infty} \frac{s_{X}\left(L, \text { type }_{1}\right)}{s_{X}\left(L, \text { type }_{2}\right)}=\frac{c\left(\text { type }_{1}\right)}{c\left(\text { type }_{2}\right)} .
$$

For example, Mirzakhani shows that nonseparating simple closed geodesics on any hyperbolic surface of genus two are six times more frequent than the separating ones.

The proof of the theorem combines methods from two domains. On the one hand, technology elaborated by Mirzakhani in [M2] allows one to compute averages over $\mathcal{M}_{q, n}$ of all kinds of counting functions of simple closed geodesics (and not only Weil-Petersson volumes). To prove asymptotic formulae for individual hyperbolic surfaces, Mirzakhani elegantly relates the counting problems to the Thurston measure on the space of measured laminations $\mathcal{M} \mathcal{L}_{g, n}$ and deduces the desired results from the ergodicity of the action of the mapping class group on $\mathcal{M} \mathcal{L}_{g, n}$ with respect to the Thurston measure, a result proved by H. Masur.

\section{The Symplectic World Witten's Conjecture}

Complex projective space $\mathbb{C} \mathrm{P}^{n}$ mentioned in the introduction carries the natural tautological line bundle: its fiber over a "point" $[\mathcal{L}] \in \mathbb{C P}^{n}$ is the line $\mathcal{L}$ considered as a vector space. Any complex line bundle $\xi$ over a compact manifold $M$ can be induced from the tautological bundle by an appropriate map $f_{\xi}: M \rightarrow \mathbb{C P}^{n}$ (for a sufficiently large $n$ depending on $M$ ). The second cohomology of the complex projective space $H^{2}\left(\mathbb{C} \mathrm{P}^{n} ; \mathbb{Z}\right) \simeq \mathbb{Z}$ has a distinguished generator $c_{1}$. The induced element $f_{\xi}^{*} c_{1} \in H^{2}(M ; \mathbb{Z})$ is called the first Chern class of the line bundle $\xi$.

There is a natural bijective correspondence between hyperbolic metrics of constant negative curvature with $n$ cusps and complex structures endowed with $n$ distinct marked points $x_{1}, \ldots, x_{n}$ on a closed smooth surface of genus $g$. In this section we use this latter interpretation of the moduli space $\mathcal{M}_{g, n}$. 
Consider the (co)tangent space $\mathcal{L}\left(C, x_{i}\right)$ to the Riemann surface $C$ at the marked point $x_{i}$. Varying $\left(C, x_{1}, \ldots, x_{n}\right)$ in $\mathcal{M}_{g, n}$ we get a family of complex lines $\mathcal{L}\left(C, x_{i}\right)$ parameterized by the points of $\mathcal{M}_{g, n}$. This family forms a line bundle $\mathcal{L}_{i}$ over the moduli space $\mathcal{M}_{g, n}$. This tautological line bundle $\mathcal{L}_{i}$ extends to the natural Deligne-Mumford compactification $\overline{\mathcal{M}}_{g, n}$ of the initial moduli space. The space $\overline{\mathcal{M}}_{g, n}$ is a nice compact complex orbifold, so for any $i=1, \ldots, n$ one can define the first Chern class $\psi_{i}:=c_{1}\left(\mathcal{L}_{i}\right)$. Recall that cohomology has a ring structure, so taking a product of $k$ cohomology classes of dimension 2 (as the first Chern class) we can integrate the resulting cohomology class over a compact complex manifold of complex dimension $k$. In particular, for any partition $d_{1}+\cdots+d_{n}=$ $3 g-3+n$ of $\operatorname{dim}_{\mathbb{C}} \overline{\mathcal{M}}_{g, n}=3 g-3+n$ into the sum of nonnegative integers, one can integrate the product $\psi_{1}^{d_{1}} \ldots \psi_{n}^{d_{n}}$ over the orbifold $\overline{\mathcal{M}}_{g, n}$. By convention, the intersection number (or the "correlator" in a physical context) is defined as

$$
\left\langle\tau_{d_{1}} \ldots \tau_{d_{n}}\right\rangle_{g}:=\int_{\overline{\mathcal{M}}_{g, n}} \psi_{1}^{d_{1}} \ldots \psi_{n}^{d_{n}} .
$$

As always, when there are plenty of rational numbers indexed by partitions or such, it is useful to wrap them into a single generating function. The resulting generating function is really famous. For physicists it is a partition function in two-dimensional quantum gravity. In mathematical terms, E. Witten conjectured in 1991 a certain recursive formula for the numbers (13) and interpreted this recursion in the form of $\mathrm{KdV}$ differential equations satisfied by the generating function. The conjecture caused an explosion of interest in the mathematical community: a single formula interlaced quantum gravity, algebraic geometry, enumerative geometry, combinatorics, topology, and integrable systems.

The first proof of Witten's conjecture is due to M. Kontsevich, who used metric ribbon graphs as a "combinatorial model" of the moduli space to express the intersection numbers (13) as a sum over 3-valent ribbon graphs. Maryam Mirzakhani suggested in [M1] an alternative proof. She ingeniously applied techniques of symplectic geometry to moduli spaces of bordered Riemann surfaces $\mathcal{M}_{g, n}\left(L_{1}, \ldots, L_{n}\right)$ discussed in the previous section. Namely, Mirzakhani recognized the intersection numbers (13) in the coefficients $C_{\alpha}$ from formula (12) for the Weil-Petersson volumes $V_{q, n}(L)$ (up to a routine normalization factor). This allowed Mirzakhani to reduce the recurrence relations for the intersection numbers contained in Witten's formula to recurrence relations for the volumes $V_{g, n}(L)$ discussed above and thus prove Witten's conjecture. (Yet other proofs are due to A. Okounkov and R. Pandharipande, who used the Gromov-Witten theory of $\mathbb{P}^{1}$ and to M. Kazarian and S. Lando, who used the ELSV-formula.)

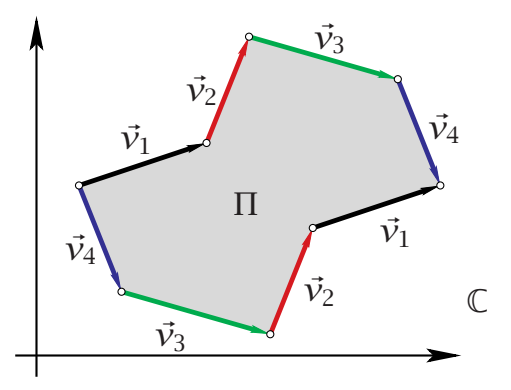

\section{Figure 1.}

\section{The Flat World Families of Translation Surfaces}

To construct a flat metric on a surface of genus different from one, we have to allow isolated conical singularities. We consider only those flat metrics which mimic a flat metric on a torus: namely, parallel transport of any tangent vector along any closed curve avoiding singularities is required to bring the vector to itself. A surface endowed with this kind of flat metric is called a translation surface. Since parallel transport along a small loop around any conical singularity brings the vector to itself, the cone angle at any singularity on a translation surface is an integer multiple of $2 \pi$.

Similar to what one finds in the case of the torus, all translation surfaces can be obtained by the following construction. Consider a collection of vectors $\vec{v}_{1}, \ldots, \vec{v}_{n}$ in $\mathbb{R}^{2}$ and arrange these vectors into a broken line. Construct another broken line starting at the same point as the first one, arranging the same vectors in the order $\vec{v}_{\pi(1)}, \ldots, \vec{v}_{\pi(n)}$, where $\pi$ is some permutation of $n$ elements. By construction the two broken lines share the same endpoints; suppose that they bound a polygon as in Figure 1. Identifying the pairs of sides corresponding to the same vectors $\vec{v}_{j}$, $j=1, \ldots, n$, by parallel translations, we obtain a closed topological surface.

It is convenient to consider the vertical direction as part of the structure. Under this convention, the structure of a translation surface is equivalent to the structure of a pair (Riemann surface $C$, holomorphic 1-form $\omega$ on it). As a complex coordinate on $C$ one can use the coordinate $z$ in the complex plane $\mathbb{C}$ where the polygon $\Pi$ lives, the holomorphic 1-form $\omega$ is the form $d z$ in these coordinates, $\omega$ has zeroes exactly at the conical singularities, and the order of zero at a singularity with the cone angle $2 \pi\left(d_{i}+1\right)$ is $d_{i}$.

The polygon in our construction depends continuously on the vectors $\vec{v}_{i}$. This means that the topology of the resulting translation surface (its genus $g$, the number and the types of the resulting conical singularities) do not change under small deformations of the vectors $\vec{v}_{i}$. Fixing a collection of cone angles $2 \pi\left(d_{1}+1\right), \ldots, 2 \pi\left(d_{m}+1\right)$ 
with integer $d_{i}$ for $i=1, \ldots, n$, we get a family $\mathcal{H}\left(d_{1}, \ldots, d_{m}\right)$ of translation surfaces. The vectors $\vec{v}_{1}, \ldots, \vec{v}_{n}$ can be viewed as complex coordinates in this family, called period coordinates. These coordinates define an orbifold structure and a natural volume element on every $\mathcal{H}\left(d_{1}, \ldots, d_{m}\right)$.

Readers preferring algebro-geometric language may view any such family as a stratum in the moduli space $\mathcal{H}_{g}$ of pairs (Riemann surface $C$, holomorphic 1-form $\omega$ on $C$ ), where the stratum is specified by the degrees $d_{1}, \ldots, d_{m}$ of the zeroes of $\omega$.

Each stratum admits a natural action of the group $\mathrm{GL}(2, \mathbb{R})$. A linear transformation $g \in \mathrm{GL}(2, \mathbb{R})$ of the ambient $\mathbb{R}^{2} \simeq \mathbb{C}$ maps the polygon $\Pi$ to a polygon $g$. Identifying pairs of parallel sides of $g \Pi$ by translations, we get a translation surface $g \cdot S$.

The subgroup $\operatorname{SL}(2, \mathbb{R}) \subset \mathrm{GL}(2, \mathbb{R})$ preserves the flat area. This implies that the action of $\operatorname{SL}(2, \mathbb{R})$ preserves the real hypersurface $\mathcal{H}_{1}\left(d_{1}, \ldots, d_{m}\right)$ of translation surfaces of unit area. The latter codimension one subspace can be compared to the unit sphere (or rather to the unit hyperboloid) in the ambient stratum $\mathcal{H}\left(d_{1}, \ldots, d_{m}\right)$. The action of the group $\operatorname{SL}(2, \mathbb{R})$ preserves the naturally induced volume element on our "unit hyperboloid." The flow induced by the action of the diagonal subgroup is called the Teichmüller geodesic flow.

Theorem (H. Masur, W. A. Veech). For any $\left(d_{1}, \ldots, d_{m}\right)$, the stratum $\mathcal{H}_{1}\left(d_{1}, \ldots, d_{m}\right)$ has finite volume. The Teichmüller geodesic flow is ergodic on every connected component of every stratum.

Here "ergodic" means that any measurable subset invariant under the action of the group has necessarily measure zero or full measure. The ergodic theorem says that in such situations the orbit of almost every point homogeneously fills the ambient connected component. Namely, for almost all starting data the "time" average of an integrable function $f$ over the corresponding trajectory coincides with the "space" average, that is, with the integral of $f$ over the ambient connected component of $\mathcal{H}_{1}\left(d_{1}, \ldots, d_{m}\right)$.

We state the ergodic theorem for almost all starting data, because even for extremely nice and smooth maps (like a map homogeneously winding

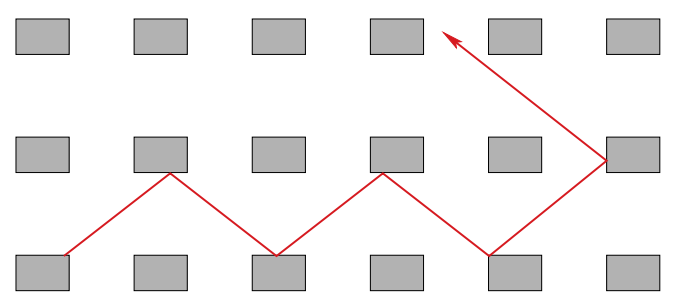

Figure 2. a circle twice around itself) certain rare trajectories behave highly nonhomogeneously, accumulating on some peculiar (often fractal) sets. Though these atypical starting data have measure zero, the multitude of types of the corresponding atypical trajectories is enormous. For example, for the map of the circle mentioned above and for almost any real number $d$ between 0 and 1 , one can find a trajectory whose closure has Hausdorff dimension $d$.

\section{The Magic Wand}

Dynamical systems for which all possible orbit closures and all possible ergodic measures are described by a short list of possible simple cases are very rare. An extremely important class of examples comes from very special dynamical systems on homogeneous spaces. The fundamental theorem of M. Ratner describing possible measures and orbit closures has had fantastic applications to number theory developed by S. G. Dani, G. Margulis, and others. The theorem below shows that though the moduli space is not a homogeneous space, it is almost one.

Theorem ([EM], [EMM]). The closure $\mathcal{L}$ of any $\mathrm{GL}(2, \mathbb{R})$-orbit in any stratum $\mathcal{H}\left(d_{1}, \ldots, d_{m}\right)$ is a complex orbifold given locally by linear equations in period coordinates.

Every $\mathrm{SL}(2, \mathbb{R})$-orbit closure supports a unique ergodic $\mathrm{SL}(2, \mathbb{R})$-invariant probability measure, and any ergodic $\mathrm{SL}(2, \mathbb{R})$-invariant measure is supported on a suborbifold.

As vague conjectures (or better, very optimistic dreams), these properties were discussed for a long time without the slightest hint of a general proof. The only exception is the case of surfaces of genus two, for which ten years ago C. McMullen completely classified all possible orbit closures. Applying clever geometric construction, he managed to reduce the problem to genus one, where Ratner's theorem becomes applicable.

The proof of A. Eskin, M. Mirzakhani, and A. Mohammadi is a titanic work which took many years. It absorbed numerous fundamental developments in dynamical systems which mostly do not have any direct relation to moduli spaces. To mention only a few, it incorporates certain ideas of the low entropy method of M. Einsiedler, A. Katok, E. Lindenstrauss; results of G. Forni and of M. Kontsevich on the Lyapunov exponents of the Teichmüller geodesic flow; the ideas from the work of Y. Benoit and J.-F. Quint on stationary measures; iterative improvement of the properties of the invariant measure inspired by the approach of G. Margulis and G. Tomanov to the actions of unipotent flows on homogeneous spaces; some fine ergodic results due to Y. Guivarch and A. Raugi.

In some situations the theorem really serves as a Magic Wand. Consider, for example, the windtree 
model introduced by physicists P. and T. Ehrenfest more than a century ago. We study the billiard in the plane filled periodically with the identical rectangular obstacles as in Figure 2. A trajectory might go far away, then return relatively close back to the starting point, then make other long trips. The diffusion rate $v$ describes the average rate $T^{v}$ with which the trajectory expands in the plane on a long range of time $T \gg 1$. More formally,

$v:=\lim _{T \rightarrow \infty} \frac{\log \text { (diameter of trajectory of length } T)}{\log T}$.

For the random walk in the plane or for a billiard with periodic circular obstacles, the diffusion rate is known to be $1 / 2$ : the most distant point of a piece of trajectory corresponding to segment of time $[0, T]$ would be located roughly at a distance $\sqrt{T}$. It was recently discovered in [DHL] that for the windtree model as in Figure 2 the diffusion rate is $2 / 3$.

Suppose now that we want to find the diffusion rate for a generalized windtree model with periodic scatterers of the shape of a more complicated rational polygon. Replace the periodic billiard with an associated compact flat surface. Touch it with the Magic Wand of Eskin-Mirzakhani-Mohammadi and find its $\mathrm{SL}(2, \mathbb{R})$-orbit closure in the space of flat surfaces. Run the geodesic flow to compute the mean monodromy (Lyapunov exponents) of the appropriate block of the complex Hodge bundle, and we get the answer.

To be honest, in full generality, this strategy is a new dream (though in some situations it already works; see [DZ]). We do not yet have a classification of SL(2, $\mathbb{R}$ )-invariant orbifolds except in genus two. This presents a new challenge, which might be full of mysteries and marvels, as indicated by recent results of M. Mirzakhani and A. Wright, who have found a GL(2, $\mathbb{R})$-invariant suborbifold of completely enigmatic origin in the family $\mathcal{H}(6)$.

A billiard in a polygon is just an elegant way to describe a certain class of dynamical systems; the same kind of dynamical systems appear in solid-state physics, in conductivity theory, in the theory of surface foliations, and the Magic Wand is extremely useful for the related problems in these areas (see [Zor] for details on applications of the Magic Wand). It also opens a new way to study moduli spaces.

\section{Acknowledgments}

The author thanks A. Jackson, J. Athreya, A. Eskin, G. Forni, M. Kontsevich, H. Masur, C. McMullen, A. Wright, P. Zograf, and D. Zvonkine, whose suggestions helped to improve the presentation.

\section{References}

[DHL] V. Delecroix, P. Hubert, and S. LeliëVRE, Diffusion for the periodic wind-tree model, Ann. de l'ENS 47:6 (2014), 1085-1110.
[DZ] V. DelecroiX and A. ZORICH, Cries and whispers in wind-tree forests, arXiv:1502.06405(2015), 1-27.

[EM] A. ESKIN and M. MIRZAKHANI, Invariant and stationary measures for the $\operatorname{SL}(2, \mathbb{R})$ action on moduli space, arXiv:1302.3320(2013), 1-172.

[EMM] A. EsKIN, M. MiRZAKHANI, and A. MOHAMMADI, Isolation, equidistribution, and orbit closures for the $\operatorname{SL}(2, \mathbb{R})$ action on Moduli space, to appear in Annals of Math.

[Kl] E. KLARREICH, A tenacious explorer of abstract surfaces, Quanta Magazine, August 12, 2014; www. simonsfoundation.org/quanta/20140812-a-tenaciousexplorer-of-abstract-surfaces/

[McM] C. McMullen, The mathematical work of Maryam Mirzakhani, Proceedings of the ICM (Seoul 2014), vol. 1, Kyung Moon SA Co. Ltd., 2014, pp. 73-79; www. icm2014.org/en/vod/proceedings

[M1] M. MIRZAKHANI, Weil-Petersson volumes and intersection theory on the moduli space of curves, J. Amer. Math. Soc. 20 (2007), no. 1, 1-23.

[M2]___, Simple geodesics and Weil-Petersson volumes of moduli spaces of bordered Riemann surfaces, Invent. Math. 167 (2007), no. 1, 179-222.

[M3] _ Growth of the number of simple closed geodesics on hyperbolic surfaces, Annals of Math. (2) 168 (2008), no. 1, 97-125.

[MZg] M. MIRZAKHANI and P. ZOGRAF, Towards large genus asymptotics of intersection numbers on moduli spaces of curves, to appear in GAFA.

[W] A. WRIGHT, From rational billiards to dynamics on moduli spaces, Bull. Amer. Math. Soc. (N.S.) 53 (2016), no. $1,41-56$.

[Zor] A. ZoRICH, Le théorème de la baguette magique de A. Eskin et M. Mirzakhani, Gazette des mathématiciens 142 (2014), 39-54; English translation: arXiv: 1502.05654 